\title{
On the value of the critical point in fractal percolation
}

\author{
Damien G White
}

\begin{abstract}
We derive a new lower bound $p_{c}>0.8107$ for the critical value of Mandelbrot's dyadic fractal percolation model. This is achieved by taking the random fractal set (to be denoted $A_{\infty}$ ) and adding to it a countable number of straight line segments, chosen in a certain (non-random) way as to simplify greatly the connectivity structure. We denote the modified model thus obtained by $C_{\infty}$, and write $C_{n}$ for the set formed after $n$ steps in its construction. Now it is possible, using an iterative technique, to compute the probability of percolating through $C_{n}$ for any parameter value $p$ and any finite $n$. For $p=0.8107$ and $n=360$ we obtain a value less than $10^{-5}$; using some topological arguments it follows that 0.8107 is subcritical for $C_{\infty}$ and hence (since $C_{\infty}$ dominates $A_{\infty}$ ) for $A_{\infty}$.
\end{abstract}

\section{A new lower bound via a new model}

The dyadic fractal percolation model [5] can be described informally as follows. Fix $0 \leq$ $p \leq 1$. Divide the unit square $I=[0,1]^{2}$ into 4 equal smaller squares, and in the natural way retain each of these squares with probability $p$, or else remove it with probability $1-p$. Iterate this procedure (suitably scaled) of subdivision and random removal on each of the retained squares; in this way we obtain a nested sequence $A_{0}(\equiv I), A_{1}, A_{2}, \ldots$ of random (compact) subsets of $I$. The intersection of this sequence, which we shall denote $A_{\infty}$, is a random fractal set. For $0 \leq n \leq \infty$ let $\theta_{n}=\theta_{n}(p)$ denote the probability that there is a left-right crossing of $A_{n}$ in $I$, that is, that there is a connected component of $A_{n}$ that intersects both the left side $\{0\} \times[0,1]$ and the right side $\{1\} \times[0,1]$ of the unit square. It is well known (see [1], [3]) that there is a critical value $p_{c}$, with $0<p_{c}<1$, such that $\theta_{\infty}(p)$ is zero if and only if $p<p_{c}$. In particular, $\theta_{\infty}(p)$ is discontinuous at $p_{c}$. 


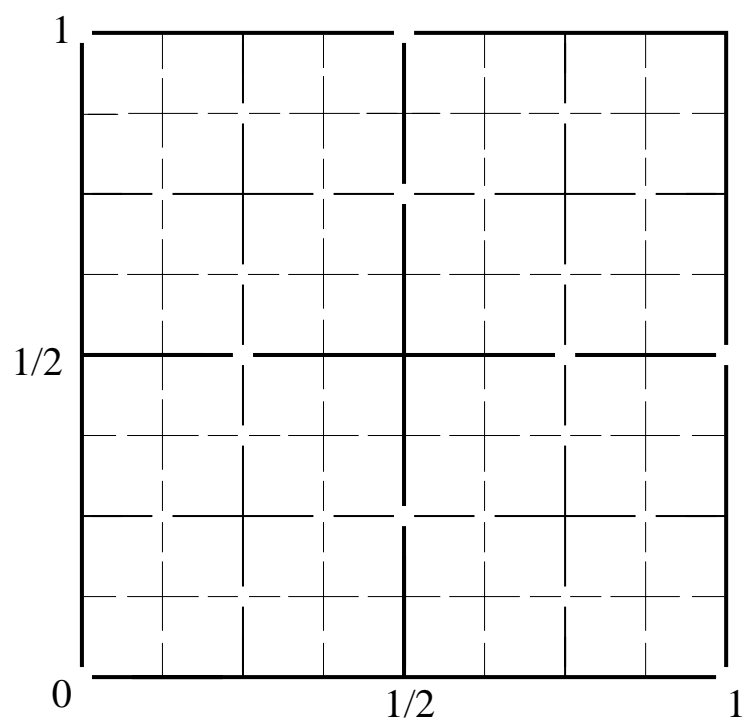

Figure 1: The set $F$ (not in full detail).

It has so far proved intractable to find the exact value of $p_{c}$. In this article we introduce a deterministic set $F \subset I$, such that the random fractal process $C_{n}=A_{n} \cup F$ has a critical value $p_{c}^{\prime}$ that can actually be computed with the help of a fairly simple computer program, giving the value $p_{c}^{\prime}=0.811$ (to three decimal places). As far as rigorous proofs are concerned, we prove (with the aid of the computer) that $p_{c}^{\prime}>0.8107$; moreover, since $C_{n}$ dominates $A_{n}$ for all $n$, it will follow that 0.8107 is a lower bound for the classical critical point $p_{c}$. This improves on the previous lower bound of $1 / \sqrt{2} \approx 0.7071$ [1].

A precise definition of $F$ is postponed to Section 3, and for now the reader is simply referred to Figure 1. Observe that there are exactly 5 connected components of $F$ that intersect the boundary $\partial I$ of the unit square. (See Section 3 for a justification of this statement.) Since for all $n=0, \ldots \infty C_{n} \supset F$, it follows that the number of connected components of $C_{n}$ that intersect $\partial I$ is less than or equal to five; we shall say that $C_{n}$ is wired if this number is strictly less than five. Thus when $C_{n}$ is wired, connections have been formed in $A_{n}$ so that some of the five boundary components of $F$ become linked. For given $0 \leq p \leq 1$ and $n<\infty$, write $\theta_{n}^{\prime}=\theta_{n}^{\prime}(p)$ for the probability that $C_{n}$ is wired. Since $A_{n} \subset C_{n}$, if there is a left-right crossing in $A_{n}$ then $C_{n}$ is certainly wired, so $\theta_{n}^{\prime}(p) \geq \theta_{n}(p)$ for all $p$ and all finite $n$. Moreover the sequence $\left(C_{n}\right)$ is nested, so $\theta_{n}^{\prime}(p)$ is nonincreasing in $n$ and we may define $\theta_{\infty}^{\prime}(p)=\lim _{n \rightarrow \infty} \theta_{n}^{\prime}(p)$.

A note on probability measures: we prefer to think of our underlying probability space in such a way that the underlying probability measure itself depends on the parameter $p$, so 
the sequence $\left(A_{n}\right)$ depends on $p$ only through its induced measure (or law). The law of $\left(A_{n}\right)$ will be denoted $\mathbf{P}_{p}$. Thus $\theta_{n}^{\prime}(p)=\mathbf{P}_{p}\left(C_{n}\right.$ is wired $)$ for $n$ finite, and $\theta_{\infty}^{\prime}(p)=\lim _{n \rightarrow \infty} \mathbf{P}_{p}\left(C_{n}\right.$ is wired).

We have found it convenient to take what might be called a physicist's approach, in defining $\theta_{\infty}^{\prime}$ without direct reference to the infinite model $C_{\infty}$. A more common approach for a mathematician might have been to define $\theta_{\infty}^{\prime}(p)$ to be $\mathbf{P}_{p}\left(C_{\infty}\right.$ is wired), in analogy with the definition of $\theta_{\infty}(p)$ above. In fact, the two approaches are equivalent, as we see from the following proposition.

Proposition 1 We have $\left\{C_{\infty}\right.$ is wired $\}=\bigcap_{n \geq 0}\left\{C_{n}\right.$ is wired $\}$. In particular, for all $0 \leq$ $p \leq 1, \mathbf{P}_{p}\left(C_{n}\right.$ is wired $)$ converges to $\mathbf{P}_{p}\left(C_{\infty}\right.$ is wired) as $n \rightarrow \infty$.

The corresponding result for the $A_{n}$ is well-known, being a simple consequence of the $A_{n}$ being nested compact sets [6], and indeed yields $\theta_{\infty}(p)=\lim _{n \rightarrow \infty} \theta_{n}(p)$. The $C_{n}$ are however not in general compact, and the proof of Proposition 1 involves a considerable amount of topological work. As the proposition will in any case not be used in this paper we omit the proof (but see [7]).

On the other hand, the next proposition (proved in Section 5) will be crucial to what follows.

Proposition 2 Suppose $0<p<1$ with $\theta_{\infty}^{\prime}(p)>0$. Then

$$
\theta_{\infty}^{\prime}(p) \geq \frac{p^{-n}-3}{2^{2 n+3}}
$$

for all $n \geq 1$.

An important consequence of Proposition 2 is

Proposition 3 There is a first order phase transition in $\theta_{\infty}^{\prime}$, that is, there exists $p_{c}^{\prime}$ with $0<p_{c}^{\prime}<1$ such that $\theta_{\infty}^{\prime}(p)$ is zero if and only if $p<p_{c}$.

Proof By a simple coupling argument, $\theta_{n}^{\prime}(p)$ is nondecreasing in $p$ and nonincreasing in $n$; moreover, $\theta_{n}^{\prime}$ is continuous when $n$ is finite, since $C_{n}$ depends on only finitely many Bernoulli random variables. It therefore follows that $\theta_{\infty}^{\prime}$ is nondecreasing and right-continuous.

Set $p_{c}^{\prime}=\inf \left\{p: \theta_{\infty}^{\prime}(p)>0\right\}=\sup \left\{p: \theta_{\infty}^{\prime}(p)=0\right\}$. Since

$$
\theta_{\infty}^{\prime}(p)=\lim _{n \rightarrow \infty} \theta_{n}^{\prime}(p) \geq \lim _{n \rightarrow \infty} \theta_{n}(p)=\theta_{\infty}(p),
$$


we have $p_{c}^{\prime} \leq p_{c}<1$. On the other hand, by elementary branching process theory $A_{\infty}=\emptyset$ $\left(\mathbf{P}_{p}\right.$-a.s. $)$ for $p \leq 1 / 4$, so it follows that $p_{c}^{\prime} \geq 1 / 4>0$. Finally, $\theta_{\infty}^{\prime}\left(p_{c}^{\prime}\right)>0$ since from Proposition 2 and right-continuity we have $\theta_{\infty}^{\prime}\left(p_{c}^{\prime}\right)>2^{-2 n-3}\left(p_{c}^{\prime-n}-3\right)$ for all $n$.

To make calculations about $\left(C_{n}\right)$ we shall want to be able to express $\theta_{n+1}^{\prime}$ as a function of $\theta_{n}^{\prime}$. This cannot be done directly: first we need to break down the event ' $C_{n}$ is wired' into various sub-events, in other words, to be more specific about the different ways the event ' $C_{n}$ is wired' can occur. Label the five connected components of $F \cap \partial I$ by $S_{1}, \ldots S_{5}$ as in Figure 2. For $0 \leq n \leq \infty$ we define the (random) equivalence relation $\stackrel{n}{\sim}$ on the numbers 1 to 5 by saying that $i \stackrel{\sim}{\sim} j$ if and only if $S_{i}$ and $S_{j}$ are contained in the same connected component of $C_{n}$. Thus $C_{n}$ is wired when there exist $i \neq j$ such that $i \sim j$; that is, $\left\{C_{n}\right.$ is wired $\}=\{\stackrel{n}{\sim} \neq$ equality $\}$. Hence,

$$
\theta_{n}^{\prime}(p)=1-\mathbf{P}_{p}(\stackrel{n}{\sim}=\text { equality })
$$

Note that not all equivalence relations are geometrically realisable: for example, there is no realisation of $C_{n}$ for which $\stackrel{n}{\sim}$ is the relation with equivalence classes $\{1,2,4\},\{3,5\}$ (see Figure 2).

Let $E$ be the set of all possible equivalence relations on the numbers 1 to 5 . For $n \geq 0$ and $0 \leq p \leq 1$, let $\pi_{n}(p)$ be the probability vector indexed by $E$ with $\pi_{n}^{e}(p)=\mathbf{P}_{p}(\stackrel{n}{\sim}=e)$. In Section 4 we give a constructive proof of the following:

Proposition 4 There exists a function $f_{p}:[0,1]^{E} \rightarrow[0,1]^{E}$ with the property that $\pi_{n}(p)=$ $f_{p}\left(\pi_{n-1}(p)\right)$ for all finite $n \geq 1$. This function can be written down explicitly.

The explicit formulation of $f_{p}$ is extremely complicated so for reasons of space will not be given here.

For different values of $p$, we used Mathematica to iterate $f_{p}$ a large number $(360)$ of times starting from $\pi_{0}(p)$. (Since $A_{0} \equiv I, \pi_{0}(p)$ is the vector with unit mass on the total equivalence relation, that is, on the relation with one equivalence class $\{1, \ldots 5\}$.) Using (1) we were thus able to compute $\theta_{360}^{\prime}(p)$. Of particular interest are the values $\theta_{360}^{\prime}(0.8107)=2.179 \times 10^{-6}$ and $\theta_{360}^{\prime}(0.8115)=0.9659$. These results strongly suggest that $p_{c}^{\prime}=0.811$ (to three decimal places), however we have yet found no way of proving that $\theta_{n}^{\prime}(0.8115)$ remains bounded away from zero for all $n$.

To prove that $\theta_{n}^{\prime}(0.8107)$ does converge to zero is straightforward: applying Proposition 2 with $p=0.8107$ and $n=6$ we see that $p_{c}^{\prime}<0.8107$ implies $\theta_{\infty}^{\prime}(0.8107)>1.595 \times 10^{-5}$; but this would be in contradiction with the computed value of $\theta_{360}^{\prime}(0.8107)$. 
Since (from the proof of Proposition 3) $p_{c}^{\prime} \leq p_{c}$, we have therefore proved

Theorem 50.8107 is a lower bound for $p_{c}$ and $p_{c}^{\prime}$.

The computational results above have been double-checked by Kenery Oron, a student at the University of Utrecht, who independently implemented the algorithms described here in JAVA, without reference to the author's original Mathematica programs. Listings of the Mathematica programs, together with complete details of the function $f_{p}$, are available in Notebook form from the author on request.

The rest of this paper is concerned with covering the three points left undone in Section 1: a precise definition of the set $F$, and proofs of Propositions 2 and 4. In Section 2 we first discuss a toy model that incorporates in a fairly elementary way most of the important ideas we shall need later on, at the same time introducing some useful notation. These ideas will then be built on in Section 3, where we give a precise definition of the set $F$ and discuss some of its consequences for self-similarity properties in the new model $\left(C_{n}\right)$. Having clearly defined $F$ we are then in a position to prove Propositions 2 and 4 : Proposition 4 is proved in Section 4 and Proposition 2 in Section 5.

\section{A simple example}

Before defining the model $\left(C_{n}\right)$ itself, we briefly discuss a toy model that incorporates in a fairly elementary way most of the important ideas concerning $\left(C_{n}\right)$.

Afficianados of fractal percolation will probably be well acquainted with the 'primitive' fractal percolation model called Diamond Percolation [2, 4]. This is usually defined as follows. Begin with a graph consisting of four bonds, as in Figure 3. We call this graph a diamond. Now perform independent bond percolation with parameter $p$ on the diamond, and let $\theta_{1}^{\diamond}(p)=\mathbf{P}_{p}$ (there is an open path in the diamond from point $L$ to point $R$ ) $=$ $2 p^{2}-p^{4}$. Next, replace each open bond with a new diamond, and iterate the whole process (see Figure 4 ). In diamond percolation we are interested in the probability $\theta_{n}^{\diamond}(p)$ that there is an open path from $L$ to $R$ after $n$ iterations of the process. It is not difficult to see that these probabilities satisfy the recursion

$$
\theta_{n}^{\diamond}(p)=2\left(p \theta_{n-1}^{\diamond}(p)\right)^{2}-\left(p \theta_{n-1}^{\diamond}(p)\right)^{4}
$$

This enables us easily to compute the value of $\theta_{n}^{\diamond}(p)$ for any given $p$ and $n<\infty$. Using elementary methods (see for example Lemma 3.4 of [3]) we can even arrive at an expression 


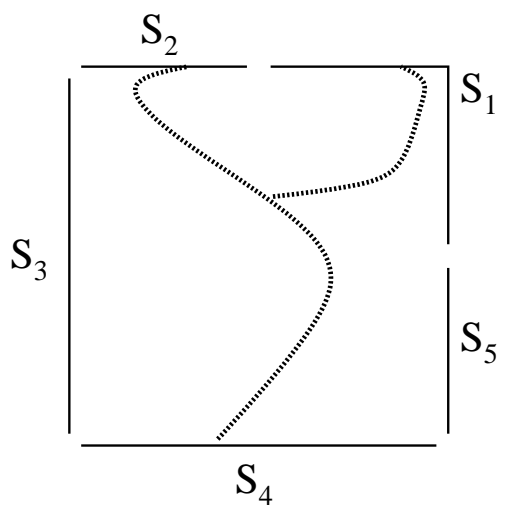

Figure 2: The five components $S_{1}, \ldots S_{5}$ of $F \cap \partial I$. In this diagrammatic representation of a realisation of $C_{n}$ (for some $n$ ) we see that $S_{1}, S_{2}$ and $S_{4}$ all lie in the same connected component of $C_{n}$ and thus $1 \stackrel{n}{\sim} 2 \stackrel{n}{\sim} 4$. Note that it would not be possible for $\stackrel{n}{\sim}$ to have equivalence classes $\{1,2,4\}$ and $\{3,5\}$ : if $A, A^{\prime}$ are connected subsets of $C_{n}$ with $A \supset$ $S_{1} \cup S_{2} \cup S_{4}$ and $A^{\prime} \supset S_{3} \cup S_{5}$, then $A \cap A^{\prime}$ is nonempty.

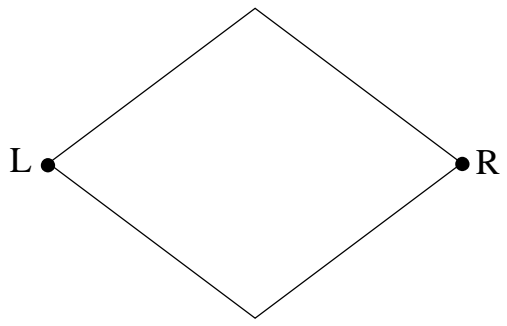

Figure 3: A diamond is a simple graph with two vertices distinguished as shown. 
for the critical point of the model

$$
p_{c}^{\diamond}=\sup \left\{p: \theta_{n}^{\diamond}(p) \rightarrow 0 \text { as } n \rightarrow \infty\right\}=\frac{3}{4} \sqrt{\frac{3}{2}} \approx 0.9186 .
$$

It is possible to formulate the diamond percolation model in terms of Mandelbrot's fractal percolation process $\left(A_{n}\right)$, as we shall now explain. First, a little notation. For $0 \leq n<\infty$, we say $Q \subset I$ is a level-n square if $Q$ is of the form $\left[a 2^{-n},(a+1) 2^{-n}\right] \times$ $\left[b 2^{-n},(b+1) 2^{-n}\right]$ for some nonnegative integers $a, b<2^{n}$. Thus $A_{n}$ is always a union of some level- $n$ squares. $Q$ is a dyadic square if $Q$ is a level- $n$ square for some $n$. For $-1 \leq i<\infty$, set $\mathbf{D}_{i}=\left\{x \in[0,1]: 2^{i} x \in \mathbf{Z}\right\}$, and let $\mathbf{D}=\bigcup_{-1}^{\infty} \mathbf{D}_{i}$ be the set of all dyadic numbers in $[0,1]$. We define $\phi: \mathbf{D} \rightarrow \mathbf{Z}$ by $\phi(x)=\min \left\{n \geq-1: x \in \mathbf{D}_{n}\right\}$. Thus for example $\phi(0)=-1, \phi(1)=0, \phi(5 / 8)=3$.

Now consider the 'grid'

$$
G:=(\mathbf{D} \times[0,1]) \cup([0,1] \times \mathbf{D}) .
$$

This set exhibits a lot of self-similarity, indeed, for any dyadic square $I_{\star}, G \cap I_{\star}$ is similar to $G$. It would however be of little interest to consider the random set $A_{n} \cup G$ as a percolation model, since this is connected for every realisation of $A_{n}$. Instead, we take the following approach. Partition $G$ into a set made up of vertical line segments

$$
V=(\mathbf{D} \times[0,1]) \backslash\left\{(x, y) \in \mathbf{D}^{2}: \phi(x) \geq \phi(y)\right\}
$$

and a set made up of horizontal line segments

$$
H=([0,1] \times \mathbf{D}) \backslash\left\{(x, y) \in \mathbf{D}^{2}: \phi(x)<\phi(y)\right\}
$$

(see Figure 5). Define now a new random set

$$
\Delta_{n}=\left(A_{n} \cup V\right) \backslash H
$$

for $0 \leq n \leq \infty$, and for $n<\infty$ define

$$
\theta_{n}^{\Delta}(p)=\mathbf{P}_{p}\left(\text { there is a left-right crossing of } \Delta_{n} \text { in } I\right) .
$$

$\Delta_{n}$ has a useful property which enables us to express $\theta_{n}^{\Delta}(p)$ in terms of simple events defined on the level-1 squares $I_{1}, \ldots I_{4}$ (as given in Figure 6). For all $n$,

$$
(0,1) \times\{1 / 2\} \subset H \subset \Delta_{n}^{c}
$$




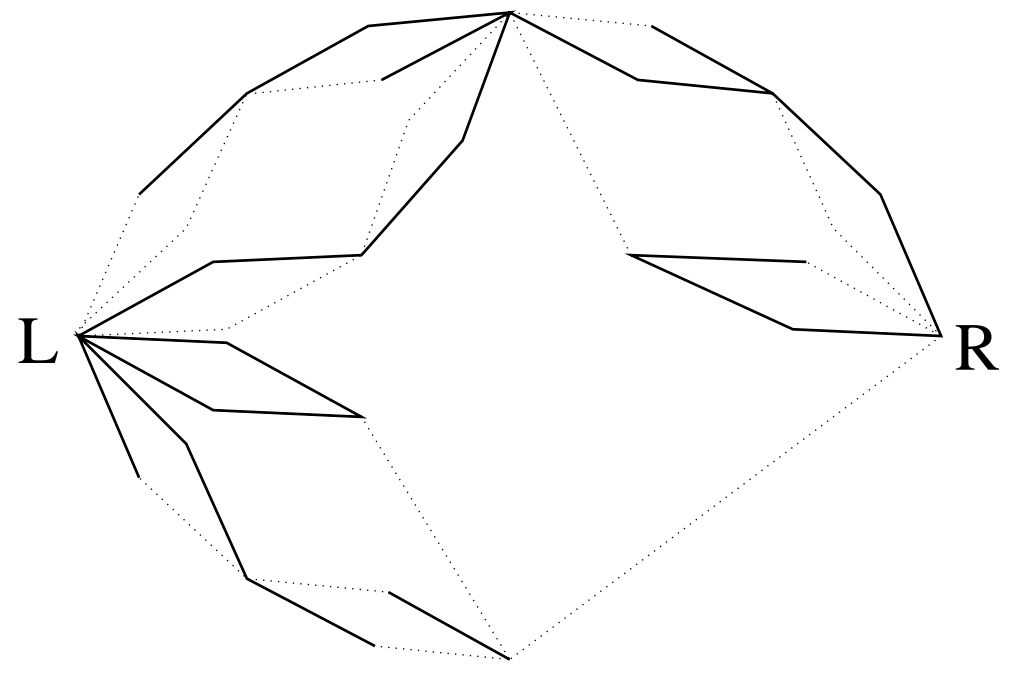

Figure 4: A possible realisation of the diamond percolation process after three iterations. The solid lines represent open bonds and the broken lines closed bonds.

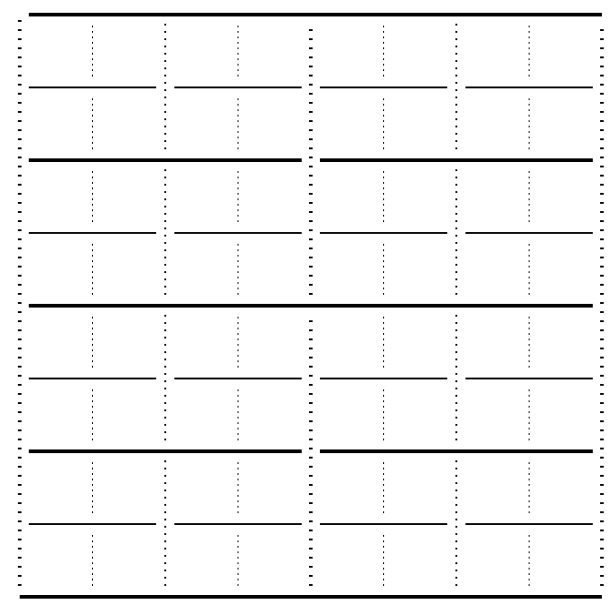

Figure 5: Part of the sets $H$ (in solid lines) and $V$ (in broken lines).

\begin{tabular}{|l|l|}
\hline $\mathrm{I}_{2}$ & $\mathrm{I}_{1}$ \\
\hline $\mathrm{I}_{3}$ & $\mathrm{I}_{4}$ \\
\hline
\end{tabular}

Figure 6: The four level-1 squares $I_{1}, \ldots I_{4}$. 
and

$$
\{1 / 2\} \times((0,1 / 2) \cup(1 / 2,1)) \subset V \subset \Delta_{n}
$$

This implies that

$$
\theta_{1}^{\Delta}(p)=\mathbf{P}_{p}\left(I_{1} \cup I_{2} \subset A_{1} \text { or } I_{3} \cup I_{4} \subset A_{1}\right)=2 p^{2}-p^{4}
$$

and in general that there is a left-right (L-R) crossing of $\Delta_{n}$ in $I$ if and only if either $(i)$ there is a L-R crossing of $\Delta_{n} \cap I_{1}$ in $I_{1}$ and of $\Delta_{n} \cap I_{2}$ in $I_{2}$ or (ii) there is a L-R crossing of $\Delta_{n} \cap I_{3}$ in $I_{3}$ and of $\Delta_{n} \cap I_{4}$ in $I_{4}$. (Note that it would not be possible to write down a similar characterisation in the classical model $\left(A_{n}\right)$ - see Figure 7.) By self-similarity in $\Delta_{n}$ we see that $\mathbf{P}_{p}\left(\mathrm{~L}-\mathrm{R}\right.$ crossing of $\Delta_{n} \cap I_{i}$ in $\left.I_{i}\right)=p \theta_{n-1}^{\Delta}$ for all $n \geq 1$ and $i=1, \ldots 4$, hence, $\theta_{n}^{\Delta}$ satisfies the recursion

$$
\theta_{n}^{\Delta}(p)=2\left(p \theta_{n-1}^{\Delta}(p)\right)^{2}-\left(p \theta_{n-1}^{\Delta}(p)\right)^{4}
$$

Of course this comes down to the same calculation as in the first paragraph of this section. Thus $\theta_{n}^{\Delta} \equiv \theta_{n}^{\diamond}$ for all $n$. In particular, the critical point for our model $\left(\Delta_{n}\right)$ is known explicitly, having the value $p_{c}^{\Delta}=\frac{3}{4} \sqrt{\frac{3}{2}}$.

\section{The model}

The model $\left(C_{n}\right)$ is defined using the dyadic grid $G$ and the function $\phi$, in a way reminiscent of the definition of $\Delta_{n}$ in the previous section. Let

$$
K=\{(0,0)\} \cup\left\{(x, y) \in \mathbf{D}^{2}:|\phi(x)-\phi(y)|=1\right\}
$$

and set $F=G \backslash K$. (See Figure 1.) We now define $C_{n}=A_{n} \cup F$ for $0 \leq n \leq \infty$.

Before proceeding, a brief remark on connected components of $F$ : notice that the lines $y=2 x$ and $y=x / 2$ do not intersect $F$, so $F$ is certainly disconnected. Indeed, the set $Y$ of Figure 8, made up of ten straight line segments of slope $2^{ \pm 1}$, satisfies $Y \cap F=\emptyset$. By

inspection, it follows that there are exactly five connected components of $F$ that intersect $\partial I$, as was stated in Section 1.

We shall see that $C_{n}$ and $\Delta_{n}$ have many similarities, but note one crucial difference. Since $\Delta_{n}$ is obtained from $A_{n}$ by adding some lines and removing others, in general we have neither $\Delta_{n} \subset A_{n}$ nor $A_{n} \subset \Delta_{n}$, and so no direct comparison is possible between $p_{c}^{\Delta}$ and $p_{c}$. On the other hand, as we saw in Section $1, A_{n}$ is a subset of $C_{n}$ and therefore the critical point for the $C_{n}, p_{c}^{\prime}$, forms a lower bound for $p_{c}$. 


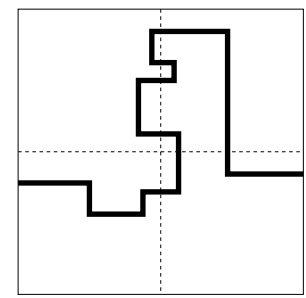

Figure 7: In this realisation of $A_{n}$ (for a given finite $n$ ), the existence of a left-right crossing is guaranteed by complicated interconnections across the boundaries of the level-1 squares. To write down all possible ways such interconnections can occur is practically speaking impossible.

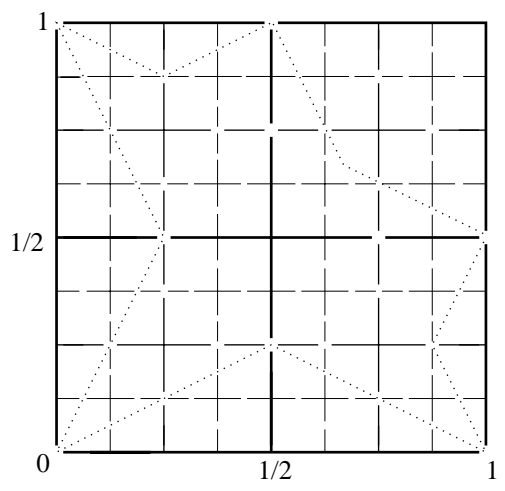

Figure 8: The set $Y$ (in broken lines) does not intersect $F$ (solid lines). 
An important similarity between $C_{n}$ and $\Delta_{n}$ is the way connection probabilities are expressable in terms of fairly simple events defined on the level-1 squares $I_{1}, \ldots I_{4}$. We have seen already how this property in $\Delta_{n}$ can be used to obtain a simple recursion equation for $\theta_{n}^{\Delta}$ : the same idea will be used (in a more sophisticated way) to prove Proposition 4 in Section 4.

In this section however we concentrate on another point in common between $C_{n}$ and $\Delta_{n}$ : what might be called stochastic self-similarity. This was rather skirted over in our treatment of $\Delta_{n}$, but for $C_{n}$ a more detailed discussion will be necessary. Stochastic selfsimilarity in $\Delta_{n}$ resulted from the simple stochastic self-similarity of the classical process $A_{n}$ together with geometric self-similarities in the deterministic sets $H$ and $V$; for $C_{n}$ we will again need the properties of $A_{n}$, now combined with self-similarities in the set $F$.

The situation for $A_{n}$ is quite simple: it is easy to see that for any positive $m, n<\infty$ and any level- $m$ square $I_{\star}$, the law of $A_{n+m} \cap I_{\star}$ conditioned on $\left\{I_{\star} \subset A_{m}\right\}$ (i.e. on ' $I_{\star}$ is retained') is identical to the law of $A_{n}$ (after scaling). Furthermore this law is invariant under rotation by any multiple of $\pi / 2$.

Turn now to $F$. Looking at Figure 1 we see straightaway that the pattern formed by $F$ within any level-1 square is remarkably similar to the set $F$ itself. To formalise this somewhat, define $J=\{(1,1),(0,1 / 2),(1 / 2,0)\}$ and observe that $(F \backslash J) \cap I_{i}$ is an exact copy of $F$ for $i=1, \ldots 4$, after the application of a similarity mapping $\nu_{i}$ consisting of a scaling and a rotation through a multiple of $\pi / 2$. (We omit a proof of this observation but it is not difficult, using the definitions at the beginning of this section.) By induction, we deduce that for any $m$ with $1 \leq m<\infty$ and any level- $m$ square $I_{\star}$, there is a $J_{\star} \subset F \cap \boldsymbol{\partial} I_{\star}$ and a similarity mapping $\nu_{\star}: I_{\star} \rightarrow I$ such that $\nu_{\star}\left(\left(F \backslash J_{\star}\right) \cap I_{\star}\right)=F$. (Here $d I_{\star}$ denotes the set consisting of the four corners of $I_{\star}$.)

We can now combine these two paragraphs to obtain a stochastic self-similarity property for $C_{n}$. If $J_{0}$ is any finite subset of $F$, for $n<\infty$ we define $C_{n}^{J_{0}}$ to be the random set $A_{n} \cup\left(F \backslash J_{0}\right)$, thus $C_{n}^{\emptyset} \equiv C_{n}$. For a level-1 square $I_{i}(i=1, \ldots 4)$ we then see that the law of $\nu_{i}\left(C_{n+1}^{J} \cap I_{i}\right)$ conditioned on $\left\{I_{i} \subset A_{1}\right\}$ is the same as that of $C_{n}$. In the general set-up above of a level-m square $I_{\star}$ we have that $\nu_{\star}\left(C_{n+m}^{J_{\star}} \cap I_{\star}\right)$ on $\left\{I_{\star} \subset A_{m}\right\}$ has the same law as $C_{n}$. Let us say that $C_{n}^{J_{0}}$ is wired in $I_{\star}$ if there is a connected component of $C_{n}^{J_{0}} \cap I_{\star}$ that contains at least two distinct components of $\left(F \backslash J_{0}\right) \cap \partial I_{\star}$. Then

$$
\mathbf{P}_{p}\left(C_{n+m}^{J_{\star}} \text { is wired in } I_{\star} \mid I_{\star} \subset A_{m}\right)=\theta_{n}^{\prime}(p) \text {. }
$$

This result will be important in the proof of Proposition 2 in Section 5. 


\section{The iteration function}

We now prove Proposition 4 , that is, we show how to find a function $f_{p}:[0,1]^{E} \rightarrow[0,1]^{E}$ such that $\pi_{n}(p)=f_{p}\left(\pi_{n-1}(p)\right)$ for all finite $n \geq 1$.

Using the terminology of Section 3 , let $\approx$ be the random equivalence relation on $1, \ldots 5$ whereby $i \approx j$ if and only if $\nu_{1}^{-1}\left(S_{i}\right)$ and $\nu_{1}^{-1}\left(S_{j}\right)$ lie in the same connected component of $C_{n}^{J} \cap I_{1}$. Then by self-similarity,

$$
\mathbf{P}_{p}\left(\stackrel{n}{\approx}=e \mid I_{1} \subset A_{1}\right)=\mathbf{P}_{p}(\stackrel{n-1}{\sim}=e)=\pi_{n-1}^{e}(p)
$$

for all finite $n \geq 1$ and $e \in E$. Since $\left\{I_{1} \not \subset A_{1}\right\} \subset\{\approx=$ equality $\}$, we therefore have

$$
\mathbf{P}_{p}(\stackrel{n}{\approx}=e)=p \pi_{n-1}^{e}(p)+(1-p) \mathbf{1}(e=\text { equality })
$$

Consider now the set $C_{n} \cap I_{1}$, and let $\sim_{1}^{n} \in E$ be defined by $i \sim_{1}^{n} j$ if and only if $\nu_{1}^{-1}\left(S_{i}\right)$ and $\nu_{1}^{-1}\left(S_{j}\right)$ lie in the same connected component of $C_{n} \cap I_{1}$. We note that $(1,1) \in F$ and that $\nu_{1}^{-1}\left(S_{3}\right) \cup\{(1,1)\} \cup \nu_{1}^{-1}\left(S_{4}\right)$ is a connected set; so the sets $\nu_{1}^{-1}\left(S_{3}\right)$ and $\nu_{1}^{-1}\left(S_{4}\right)$ are always linked through $C_{n} \cap I_{1}$, that is, $3 \sim_{1}^{n} 4$ surely. Indeed, $C_{n} \cap I_{1}=\left(C_{n}^{J} \cap I_{1}\right) \cup\{(1,1)\}$, so the connected components of $C_{n} \cap I_{1}$ are the same as for $C_{n}^{J} \cap I_{1}$ except in the case when a join is made through the point $(1,1)$. That is, the equivalence classes of $\sim_{1}^{n}$ consist of any classes of $\approx$ that contain neither 3 or 4 , together with the class $\{i: i \approx 3$ or $i \approx 4\}$. This defines a function $\eta: E \rightarrow E$ such that $\sim_{1}^{n}=\eta(\approx)$, and we have

$$
\mathbf{P}_{p}\left(\sim_{1}^{n}=e\right)=\sum_{e_{0}: \eta\left(e_{0}\right)=e} \mathbf{P}_{p}(\approx=e)
$$

The above discussion can also be applied with $I_{2}, I_{3}$ or $I_{4}$ replacing $I_{1}$, to obtain random equivalence relations $\sim_{i}^{n}$ that describe the connectivity properties of $C_{n} \cap I_{i}$ for $i=2,3,4$ (see Figure 9). With equations similar to (7) and (8), we can express the law of $\sim_{i}^{n}$ as a function of the probability vector $\pi_{n-1}$. Note moreover that $\sim_{i}^{n}$ depends only on the Cantor set construction within the subsquare $I_{i}$, therefore $\sim_{1}^{n}, \sim_{2}^{n}, \sim_{3}^{n}$ and $\sim_{4}^{n}$ are independent.

We now claim that it is possible to write $\stackrel{n}{\sim}$ as a function $\zeta$ of $\sim_{1}^{n}, \sim_{2}^{n}, \sim_{3}^{n}$ and $\sim_{4}^{n}$. It follows that

$$
\pi_{n}^{e}(p)=\sum_{v \in E^{4}: \zeta(v)=e} \mathbf{P}_{p}\left(\sim_{1}^{n}=v_{1}, \sim_{2}^{n}=v_{2}, \sim_{3}^{n}=v_{3}, \sim_{4}^{n}=v_{4}\right) ;
$$

since the $\sim_{i}^{n}$ are independent, and we can express each term $\mathbf{P}_{p}\left(\sim_{i}^{n}=v_{i}\right)$ as a function of $\pi_{n-1}$, this gives rise to the desired function $f$ satisfying $\pi_{n}=f\left(\pi_{n-1}\right)$. 
To justify the above claim, let us consider the set $L=\partial\left(I_{1}\right) \cup \ldots \cup \partial\left(I_{4}\right)$ (see Figure 10 ). This set is crucial in determining how events on the level-1 squares relate to each other, as any interconnection across the boundaries of the level-1 squares must pass through $L$. For example: in the toy model of Section 2 (formulae 3,4) we saw that $L$ is contained in $H \cup V$, where $V$ is (surely) a subset of $\Delta_{n}$ for all $n$, and $H \subset \Delta_{n}^{c}$. Thus $L \cap \Delta_{n}$ is a deterministic set; we have seen that this enables us to set up a recursion (5) for $\theta_{n}^{\Delta}$. On the other hand, we saw in Figure 7 that such a simple idea could not be applied in the classical model $A_{n}$, as here interconnections across the boundaries of the level-1 squares can occur in very complicated ways: for large $n, L \cap A_{n}$ is a complicated random set.

Here, the existence of our function $\zeta$ relies on the fact that the relationship between $C_{n}$ and $L$ is fairly simple. Namely, we can write $L \subset F \cup(K \cap L)$, knowing that $F$ is contained in $C_{n}$ (surely for all $n$ ) and $K \cap L$ is a simple finite set (as given in figure 10). Let the eight connected components of $F \cap L$ be labelled $U_{1}, \ldots U_{8}$ as in Figure 9. These components are always contained in $C_{n}$; because together they cover the crucial set $L$ (except for a few special points), we see that connections between them in $C_{n}$ are completely determined by connection events on the four level-1 squares. Formally, we define the random equivalence relation $Z$ on $1, \ldots 8$ with $i Z j$ if and only if $U_{i}$ and $U_{j}$ are contained in the same connected component of $C_{n}$ : it is possible to write $Z$ as a function of $\sim_{1}^{n}, \sim_{2}^{n}, \sim_{3}^{n}$ and $\sim_{4}^{n}$. This completes our argument, as $\stackrel{n}{\sim}$ is simply the restriction of $Z$ to $\{1, \ldots 5\}$. (See Figure 9.)

To write down $f_{p}$ we would need explicit expressions for $\zeta, \eta$, and also for the versions of $\eta$ corresponding to $I_{2}, I_{3}$ and $I_{4}$. In each case, the procedure is a straightfoward matter of processing through the combinatorial structure implied by $F$. Although the size of $E$ is too great for us realistically to do all this by hand (particularly $\zeta$ is infeasible), it is quite straightforward to translate the above argument into a computer algorithm that can be implemented in Mathematica.

\section{$5 \quad$ A bound on $\theta_{\infty}^{\prime}$}

It remains only to prove Proposition 2. To begin with: three preliminary lemmas.

We define a path to be a continuous injective map from $[0,1]$ to $I$. If $J_{0}$ is a finite subset of $F$ and $I_{\star}$ is a dyadic square, we shall say that $C_{n}^{J_{0}}=A_{n} \cup\left(F \backslash J_{0}\right)$ is path-wired in $I_{\star}$ if there is a path in $C_{n}^{J_{0}} \cap I_{\star}$ linking at least two distinct components of $\left(F \backslash J_{0}\right) \cap \partial I_{\star}$. 


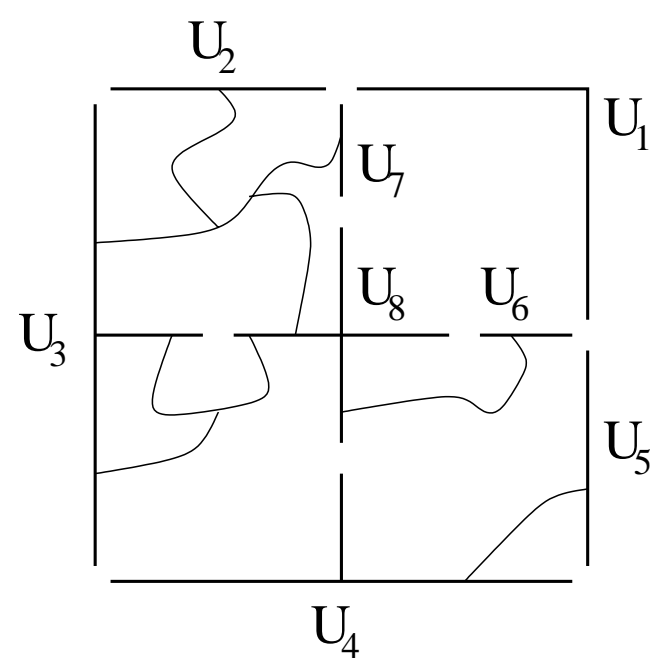

Figure 9: The eight connected components of $F \cap L$ are labelled $U_{1}, \ldots U_{8}$. Shown is a representation of a possible realisation of $C_{n}$ whereby the equivalence classes of the $\sim_{n}^{i}$ are given by $\sim_{1}^{n}:\{1\},\{2\},\{3,4\},\{5\} ; \sim_{2}^{n}:\{1,2,3,4,5\} ; \sim_{3}^{n}:\{1,2,3\},\{4,5\}$; $\sim_{4}^{n}:\{1,5\},\{2,3,4\}$. From this we can deduce that the equivalence classes of $\stackrel{n}{\sim}$ are $\{1\}$, $\{2,3\},\{4,5\}$.

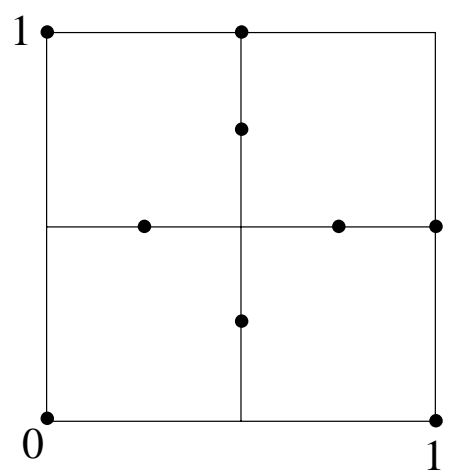

Figure 10: The straight line segments together form the set $L ; L \cap K$ consists of the nine points marked. 
Lemma 6 For any finite $J_{0} \subset F$ and any dyadic square $I_{\star}$, we have

$$
\mathbf{P}_{p}\left(C_{n} \text { is wired in } I_{\star}\right)=\mathbf{P}_{p}\left(C_{n} \text { is path-wired in } I_{\star}\right)
$$

for all $0 \leq n<\infty$ and $0 \leq p \leq 1$.

Proof First fix $I_{\star}=I$ and let $\theta_{n}^{\gamma}(p)=\mathbf{P}_{p}\left(C_{n}\right.$ is path-wired in $\left.I\right)$; we want to show that $\theta_{n}^{\prime} \equiv \theta_{n}^{\gamma}$. Now $C_{0}=I$ surely, so we know $\theta_{0}^{\prime} \equiv \theta_{0}^{\gamma} \equiv 1$. To express $\theta_{n}^{\gamma}$ in terms of $\theta_{n-1}^{\gamma}$ we can proceed in the same way as in Section 4: in fact the combinatorial arguments for $\theta_{n}^{\gamma}$ will be exactly the same as they were for $\theta_{n}^{\prime}$ and therefore we will obtain exactly the same iteration function $f$; hence it follows that $\theta_{n}^{\prime} \equiv \theta_{n}^{\gamma}$ for all finite $n$.

The lemma for general $I_{\star}$ can be proved by applying the argument of section 4 to $C_{n} \cap I_{\star}$ and the four sub-squares into which $I_{\star}$ divides.

Lemma 7 Fix $n \geq 1$ and a realisation $C_{n}$, and suppose $Q$ is some level-n square. Then for every connected component $\Sigma$ of $C_{n} \cap Q, \Sigma \cap \partial Q$ is path-connected.

Proof If $Q \subset A_{n}$ then the result is trivial (the only connected component of $C_{n} \cap Q$ is $Q$ itself) so suppose $Q \not \subset A_{n}$. It follows that $C_{n} \cap \operatorname{int}(Q) \subset F$ (where $\operatorname{int}(Q) \operatorname{denotes}$ the interior of $Q$ ). But from the discussion at the beginning of Section 3, the following is certainly clear: for every connected component $\Sigma$ of $F, \Sigma \cap \partial I$ either is empty or equals $S_{i}$ for some $i=1, \ldots 5$, and therefore is path-connected. Self-similarity of $F$ now completes the proof.

Lemma 8 Given positive finite integers $m$ and $n$ and any level $m$-square $I_{\star}$,

$$
\mathbf{P}_{p}\left(C_{n+m} \text { is wired in } I_{\star} \mid I_{\star} \subset A_{m}\right) \leq \theta_{n}(p) \text {. }
$$

Proof In the light of the discussion at the end of Section 3, the statement of the lemma is equivalent to

$$
\mathbf{P}_{p}\left(C_{n+m} \text { wired in } I_{\star} \mid I_{\star} \subset A_{m}\right) \leq \mathbf{P}_{p}\left(C_{n+m}^{J_{\star}} \text { wired in } I_{\star} \mid I_{\star} \subset A_{m}\right) .
$$

But on $\left\{I_{\star} \not \subset A_{m}\right\}$, neither $C_{n+m}$ nor $C_{n+m}^{J_{\star}}$ is wired in $I_{\star}$, so $(9)$ is the same as

$$
\mathbf{P}_{p}\left(C_{n+m} \text { wired in } I_{\star}\right) \leq \mathbf{P}_{p}\left(C_{n+m}^{J_{\star}} \text { wired in } I_{\star}\right) \text {. }
$$

By Lemma 6 this is equivalent to

$$
\mathbf{P}_{p}\left(C_{n+m} \text { path-wired in } I_{\star}\right) \leq \mathbf{P}_{p}\left(C_{n+m}^{J_{\star}} \text { wired in } I_{\star}\right) \text {. }
$$


So suppose $C_{n+m}$ is path-wired in $I_{\star}$, that is, there is a path $\gamma$ in $C_{n+m} \cap I_{\star}$ linking two distinct components of $F \cap \partial I_{\star}$. It will be sufficient for us to show that this implies the existence of a path $\gamma^{\prime}$ satisfying the same conditions as on $\gamma$ and in addition with $\gamma^{\prime} \cap J_{\star}=\emptyset$.

By considering a sub-path of $\gamma$ if necessary, we can assume $\gamma$ intersects $J_{\star}$ only at its end-points $\gamma_{0}$ and $\gamma_{1}$, if at all. (Recall that $J_{\star} \subset \delta I_{\star}$, that is, $J_{\star}$ contains only corners of $I_{\star}$; so by construction of $F$, distinct points of $J_{\star}$ are in distinct components of $F \cap \partial I_{\star}$.)

Suppose first that $\gamma_{0} \in J_{\star}$ and $\gamma_{1} \notin J_{\star}$. Then $\gamma_{0} \in \partial I_{\star}$, so there is a unique level- $(n+m)$ square $Q$ with $\gamma_{0} \in Q \subset I_{\star}$; moreover there is a point $\gamma_{Q}$ on $\gamma$, with $\gamma_{Q} \in Q \backslash\left\{\gamma_{0}\right\}$, such that the sub-path from $\gamma_{0}$ as far as $\gamma_{Q}$ is contained in $Q$; by continuity of $\gamma$ we may assume $\gamma_{Q} \in \partial Q$. Now $\gamma_{0}$ and $\gamma_{Q}$ are contained in the same connected component of $C_{n+m} \cap Q$, so by Lemma 7 there is a path $\gamma^{\star}$ in $C_{n+m} \cap \partial Q$ from $\gamma_{0}$ to $\gamma_{Q}$. Since $\gamma_{0} \in \partial I_{\star}$ we can find an $x_{0}$ on $\gamma^{\star}$ such that $x_{0} \in \partial I_{\star} \backslash J_{\star}$; let $\gamma^{\prime}$ be the path from $x_{0}$ to $\gamma_{1}$ obtained by concatening the sub-path of $\gamma^{\star}$ from $x_{0}$ to $\gamma_{Q}$ with the sub-path of $\gamma$ from $\gamma_{Q}$ to $\gamma_{1}$.

This completes the case $\gamma_{0} \in J_{\star}, \gamma_{1} \notin J_{\star}$. The three other cases are now simple to handle. If neither end-point of $\gamma$ is in $J_{\star}$, we take $\gamma^{\prime}=\gamma$; if $\gamma_{0} \notin J_{\star}$ and $\gamma_{1} \in J_{\star}$ we apply the previous argument to $\gamma_{1}$ instead of $\gamma_{0}$; if both end-points are in $J_{\star}$ we be apply the previous argument to $\gamma_{0}$ and $\gamma_{1}$.

We now proceed to prove Proposition 2, which for convenience is re-stated here.

Proposition 2 Suppose $0<p \leq 1$ with $\theta_{\infty}^{\prime}(p)>0$. Then

$$
\theta_{\infty}^{\prime}(p) \geq \frac{p^{-n}-3}{2^{2 n+3}}
$$

for all $n \geq 1$.

Proof Fix $m, n$ with $1 \leq m, n<\infty$. Consider a realisation of the process for which $C_{m+n}$ is wired. Then by inspection of Figure 8 , it follows that there exists some level-n square $I_{\star}$ with $\operatorname{int}\left(I_{\star}\right) \cap Y \neq \emptyset$, such that $C_{m+n}$ is wired in $I_{\star}$. Indeed, there exist at least two such squares, $I_{\star}$ and $I_{\star}^{\prime}$, unless $I_{\star}$ lies at one of the three corners $(0,1),(1,0)$ or $(0,0)$ of $I$. Hence, for all $0 \leq p \leq 1$,

$$
\begin{aligned}
\theta_{m+n}^{\prime}(p)= & \mathbf{P}_{p}\left(C_{m+n} \text { is wired }\right) \\
\leq & \sum \mathbf{P}_{p}\left(C_{m+n} \text { is wired in } I_{\star}\right) \\
& +\sum \mathbf{P}_{p}\left(C_{m+n} \text { is wired in } I_{\star} \text { and in } I_{\star}^{\prime}\right),
\end{aligned}
$$


where the first sum is over the three level- $n$ squares $I_{\star}$ containing either $(0,1),(1,0)$ or $(0,0)$, and the second sum is over all distinct pairs of level-n squares $I_{\star}, I_{\star}^{\prime}$ whose interiors have nonempty intersection with $Y$.

Consider the case where $I_{\star}$ and $I_{\star}^{\prime}$ are distinct. Conditioned on $\left\{I_{\star} \cup I_{\star}^{\prime} \subset A_{n}\right\}$, the event $\left\{C_{m+n}\right.$ is wired in $\left.I_{\star}\right\}$ depends only on the Cantor set construction within $I_{\star}$ (and similarly within $I_{\star}^{\prime}$ ), therefore

$$
\begin{aligned}
& \mathbf{P}_{p}\left(C_{m+n} \text { is wired in } I_{\star} \text { and in } I_{\star} \mid I_{\star} \cup I_{\star}^{\prime} \subset A_{n}\right) \\
& \quad=\mathbf{P}_{p}\left(C_{m+n} \text { is wired in } I_{\star} \mid I_{\star} \subset A_{n}\right) \mathbf{P}_{p}\left(C_{m+n} \text { is wired in } I_{\star}^{\prime} \mid I_{\star}^{\prime} \subset A_{n}\right) \\
& \quad \leq\left(\theta_{m}^{\prime}(p)\right)^{2},
\end{aligned}
$$

the inequality being an application of Lemma 8. Hence,

$$
\begin{aligned}
& \mathbf{P}_{p}\left(C_{m+n} \text { is wired in } I_{\star} \text { and in } I_{\star}^{\prime}\right) \\
& \quad=\mathbf{P}_{p}\left(I_{\star} \cup I_{\star}^{\prime} \subset A_{n}\right) \mathbf{P}_{p}\left(C_{m+n} \text { is wired in } I_{\star} \text { and in } I_{\star}^{\prime} \mid I_{\star} \cup I_{\star}^{\prime} \subset A_{n}\right) \\
& \leq p^{n}\left(\theta_{m}^{\prime}(p)\right)^{2} .
\end{aligned}
$$

Applying Lemma 8 also to the first summation in (10) we obtain

$$
\begin{aligned}
\theta_{m+n}^{\prime}(p) & \leq 3 p^{n} \theta_{m}^{\prime}(p)+\left(\begin{array}{c}
2^{n+2} \\
2
\end{array}\right) p^{n}\left(\theta_{m}^{\prime}(p)\right)^{2} \\
& \leq 3 p^{n} \theta_{m}^{\prime}(p)+2^{2 n+3} p^{n}\left(\theta_{m}^{\prime}(p)\right)^{2} .
\end{aligned}
$$

Taking the limit $m \rightarrow \infty$ we have

$$
\theta_{\infty}^{\prime}(p) \leq 3 p^{n} \theta_{\infty}^{\prime}(p)+2^{2 n+3} p^{n}\left(\theta_{\infty}^{\prime}(p)\right)^{2}
$$

which is equivalent to the statement of the lemma when $p$ and $\theta_{\infty}(p)$ are nonzero.

\section{Acknowledgments}

The initial idea for this paper came while on a visit to the Tata Institute for Fundamental Research in Mumbai, India. I would like to thank all at the Tata Institute who helped to make my visit there so highly productive and enjoyable. Thanks also to Mike Keane, Ronald Meester, Kenery Oron and Mark Orzechowski, for stimulating and useful discussions. 


\section{References}

[1] Chayes, J.T., Chayes, L. and Durrett, R. (1988) Connectivity properties of Mandelbrot's percolation process. Prob. Th. Rel. Fields 77 307-324

[2] Chayes, L. (1995) Aspects of the fractal percolation process. Progress in Prob. 37 113143

[3] Dekking, M. and Meester, R. (1990) On the structure of Mandelbrot's percolation process and other random Cantor sets. J. Stat. Phys. 58 1109-1126

[4] Machta, J. (1991) Phase transitions in fractal porous media. Phys. Rev. Lett. 66, 169172

[5] Mandelbrot, B. (1974) Intermittent turbulence in self-similar cascades: divergence of high moments and dimension of the carrier. J. Fluid Mech. 62 331-358

[6] Orzechowski, M. (1997) Geometrical and topological properties of fractal percolation. Ph.D. Thesis, University of St Andrews

[7] White, D.G. (1999) Percolation through fractals, backbends and dynamic lily ponds. Doctoral Thesis, University of Utrecht

Mathematical Institute

University of Utrecht

PO Box 80010

3508 TA Utrecht

The Netherlands

white@math.uu.nl 